\title{
Preoperative malnutrition in patients with colorectal cancer
}

\author{
Ashna Gupta, BSc \\ Eisha Gupta, MD \\ Richard Hilsden, MD, MBA \\ Jeffry D. Hawel, MD \\ Ahmad I. Elnahas, MD, MSc \\ Christopher M. Schlachta, MDCM \\ Nawar A. Alkhamesi MD, PhD
}

Accepted January 12, 2021

\author{
Correspondence to: \\ N.A. Alkhamesi \\ Division of General Surgery \\ University Hospital \\ London Health Sciences Centre \\ 339 Windermere Rd \\ London ON N6A 5A5 \\ nalkham2@uwo.ca
}

Cite as: Can J Surg 2021 November 25; 64(6). doi: 10.1503/cjs.016820
Preoperative malnutrition in patients with colorectal cancer is associated with several postoperative consequences and poorer prognosis. Currently, there is a lack of a universal screening tool to assess nutritional status, and intervention to treat preoperative malnutrition is often neglected. This review summarizes and compares preoperative screening and interventional tools to help providers optimize malnourished patients with colorectal cancer for surgery. We found that nutritional screenings, such as the Subjectibe Global Assessment, PatientGenerated Subjective Global Assessment, Prognostic Nutritional Index, Nutrition Risk Index, Malnutrition Universal Screening Tool, Nutrition Risk Screening 2002, Nutrition Risk Score, serum albumin, and prealbumin, have all effectively predicted postoperative outcome. Physicians should consider which of these tools best fits their needs based on the their mode of assessment, efficiency, and specified parameters. Additionally, preoperative nutritional support, such as trimodal prehabilitation, modified peripheral parenteral nutrition, and N-3 fatty acid and arginine supplementation, which have also benefited patients postoperatively, ought to be implemented appropriately according to their ease of execution. Given the high prevalence of preoperative malnutrition in patients undergoing surgery for colorectal cancer, it is essential that health care providers assess and treat this malnutrition to reduce postoperative complications and length of hospital stay, and to improve prognosis to augment a patient's quality of care.

La malnutrition préopératoire chez les patients atteints d'un cancer colorectal est associée à plusieurs complications postopératoires et à un pronostic plus sombre. Il n'existe actuellement aucun outil universel d'évaluation du statut nutritionnel, et les mesures visant à corriger la malnutrition préopératoire font souvent défaut. La présente revue résume et compare les outils de dépistage et d'intervention préopératoires pour aider les professionnels à améliorer l'état des patients dénutris qui doivent subir une chirurgie pour le cancer colorectal. Nous avons constaté que le dépistage nutritionnel à l'aide de questionnaires tels que l'Évaluation globale subjective, l'Index nutritionnel pronostique, l'Outil universel de dépistage de la malnutrition, NRS 2002 (Nutrition Risk Screening 2002), l'évaluation du risque nutritionnel, et le dosage de l'albumine et de la préalbumine sériques, a permis de prédire avec justesse l'issue de la chirurgie. Les médecins devraient vérifier lequel de ces outils est le mieux adapté à leurs besoins selon leur modalité d'évaluation, leur efficience et autres paramètres spécifiques. Également, un soutien nutritionnel préopératoire, comme la préadaptation trimodale, la nutrition parentérale périphérique modifiée et les suppléments d'acides gras N-3 et d'arginine, qui ont aussi donné des résultats postopératoires favorables, devrait être appliqué selon sa facilité d'administration. Étant donné la forte prévalence de la malnutrition préopératoire chez les patients soumis à une chirurgie pour cancer colorectal, les professionnels de la santé se doivent d'évaluer et de corriger la malnutrition afin de prévenir les complications postopératoires, d'abréger la durée du séjour hospitalier, et d'améliorer ainsi le pronostic et la qualité des soins.

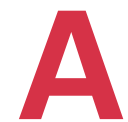
$s$ preoperative malnutrition is prevalent among patients with cancer, ${ }^{1}$ the need to assess, monitor and treat it has become increasingly important. Patients with cancer often experience nutritional decline because of the progression of the disease as well as its treatment. The etiology of malnutrition in cancer is a complex integration of physical, psychological, and social factors that influence dietary intake, metabolism and local 
gastrointestinal function. ${ }^{2}$ Consequently, cancer cachexia is common, leading to significant weight loss, weakness and sarcopenia. ${ }^{1,2}$

In particular, patients with colorectal cancer compared with other types of cancer have been found to have one of the highest rates of malnutrition due to its local effects on bowel function from obstruction and malabsorption. ${ }^{1}$ About $35 \%$ of patients undergoing colorectal surgery are moderately to severely malnourished preoperatively. ${ }^{3}$

Preoperative malnutrition among patients with colorectal cancer is associated with numerous postoperative consequences and poorer prognosis. Malnourished patients experience significant postoperative weight loss and have more frequent occurrences of septic shock as well as increased requirements for postoperative mechanical ventilation, blood transfusions, and return to the operating room. ${ }^{1}$ Malnutrition can also contribute to immunosuppression with greater occurrences of postoperative infections and inflammatory response. Additionally, micronutrient deficiencies may cause patients to have increased inflammation, lower serum albumin levels, and higher anastomotic leak rates. ${ }^{4}$ Mildly to severely malnourished patients are reported to have significantly longer hospital stays than wellnourished patients and take longer to recover gastrointestinal function. ${ }^{3}$

Despite the negative implications of malnutrition, its assessment is often neglected in preoperative health care practice. ${ }^{5}$ This is made difficult for providers because there is currently no single existing standard for assessing preoperative nutritional status that reliably encompasses all the clinically meaningful facets of malnutrition. Consequently, different means of measuring nutrition yield widely differing rates of malnutrition. For example, use of the Standard Global Assessment (SGA) has yielded a malnutrition rate of $36.4 \%$, whereas malnutrition rates among the same sample based on a body mass index (BMI) lower than 18 and triceps skinfold were $7.6 \%$ and $53 \%$, respectively. ${ }^{6}$

This increased risk of morbidity and mortality among malnourished patients with colorectal cancer represents an opportunity to improve outcomes. Several studies have previously reported data on specific individual assessments and interventions; however, a comprehensive review of this issue is lacking in the literature. A meta-analysis or systematic review was not performed owing to the variability in studies analyzing specific screening tools and an insufficient number of studies quantitatively reporting the impact of preoperative nutritional support for patients with colorectal cancer. At present, screening for malnutrition and nutritional support preoperatively are not commonly put into effect, despite published evidence demonstrating a benefit. To our knowledge, this is the first comprehensive narrative review of malnutrition screening and interventions to help oncology providers optimize patients for surgery.

\section{LITERATURE REVIEW}

We performed a literature search in PubMed including all studies published from January 1994 to June 2019 using the search terms "Preoperative malnutrition in colorectal cancer patients," "Nutritional assessments colorectal cancer," "Postoperative nutritional care colorectal cancer," "Postoperative nutritional supplementation colorectal cancer," and "Colorectal cancer postoperative nutritional care TPN." We searched additional medical subject headings $(\mathrm{MeSH})$, including "Nutrition assessment," "Nutritional status," "Nutritional screening," "Nutritional Index," "Malnutrition," "Neoplasm," "Gastrointestinal neoplasm," "Colorectal neoplasm," "Colorectal cancer," "Colorectal surgery," "Intra-abdominal surgery," and "Preoperative care." Additionally, we manually cross-referenced the searched articles to supplement our findings.

Peer-reviewed publications that were randomized controlled trials, clinical trials, reviews and retrospective studies were included. All nonhuman publications were excluded from our review. The original literature search included English language studies and publication dates from 1994 to present; however, if appropriate publication in another language or earlier studies were identified during reference review, these papers were considered for inclusion. Through our search, a total of 38 papers were included.

\section{Preoperative nutritional assessments}

To improve the nutritional status of malnourished patients with colorectal cancer, the process begins with screening. There are many screening tools reported in the literature. For example, the SGA, Patient-Generated Subjective Global Assessment (PG-SGA), Prognostic Nutritional Index (PNI), Nutritional Risk Index (NRI), Malnutrition Universal Screening Tool (MUST), Nutritional Risk Screening 2002 (NRS-2002), Reilly's Nutrition Risk Score (NRS), serum albumin, and prealbumin are a few of several nutritional assessments and markers used to screen and diagnose patients with malnutrition.

\section{Subjective Global Assessment and Patient-Generated Global Assessment}

The SGA is the current reference standard against other nutritional screening tools as it assesses a patient's nutritional status based on their medical history (weight change, dietary intake, gastrointestinal symptoms, functional capacity, and metabolic stress) and physical examination (subcutaneous fat loss, muscle wasting, edema, and ascites). ${ }^{7}$ The SGA form, which incorporates all the aforementioned characteristics, requires a trained health 
care professional to record the specific characteristics on this form. Once the medical history, physical examination, and documentation are completed, patients are then graded as SGA-A (well-nourished), SGA-B (mild to moderately malnourished), or SGA-C (severely malnourished). In a prospective observational study of 149 patients, the SGA was found to correlate with postoperative hospital stay and overall complication rates. ${ }^{3}$ Patients in the SGA-A category had significantly shorter hospital stays than those in SGA-B and SGA-C categories, as length of hospital stay increased with malnutrition severity. The overall complication rates of SGA-A, SGAB, and SGA-C were $11 \%, 31 \%$ and $40 \%$, respectively. Similarly, resumption of normal diet and time until first defecation increased significantly as the severity of malnutrition increased, suggesting that the SGA can effectively predict recovery of gastrointestinal function. ${ }^{3}$

However, because the SGA is a time-consuming assessment that must be completed entirely by a trained professional, the PG-SGA, a modified form of SGA, was developed as a useful alternative. ${ }^{8}$ Although both the SGA and PG-SGA may take up to 15 minutes to complete, the PG-SGA can be partially completed by the patient (in the form of a questionnaire), decreasing the time needed by the professional to complete the assessment. ${ }^{7}$ The PG-SGA also includes a wider range of nutritional impact symptoms, making it reliable to evaluate the nutritional status of a patient with cancer. PG-SGA scores have been found to be a strong predictor of nutritional status based on SGA, with sensitivity of $98 \%$ and specificity of $82 \%$ in identifying malnourishment in patients with cancer. ${ }^{9}$ Although the subjectivity of both the SGA and PG-SGA may constitute a disadvantage, both assessments are noninvasive and can be performed at the bedside, rendering them advantageous nutritional assessments. ${ }^{10}$

\section{Prognostic Nutritional Index}

There is a strong correlation between the preoperative PNI and postoperative complications in patients with colorectal cancer. ${ }^{11,12}$ The PNI, a measure of serum albumin concentration in conjunction with total lymphocyte count, indicates nutritional and immunological status in patients with gastrointestinal cancer. The following formula may be used to calculate PNI: $10 \times$ serum albumin $(\mathrm{g} / \mathrm{dL})+0.005 \times$ total lymphocyte count $\left(\right.$ per $\left.\mathrm{mm}^{3}\right){ }^{31}$

$\mathrm{Cao}$ and colleagues ${ }^{11}$ retrospectively categorized 228 patients with colorectal cancer according to their preoperative PNI status as either PNI-high $(\geq 44.55)$ or PNIlow $(<44.55)$. They assessed postoperative complications following curative laparoscopic surgery. Postoperative complications were reported in $23.5 \%$ of the PNI-low group, compared with $7.29 \%$ in the PNI-high group. Moreover, the rate of severe postoperative complications, defined as requiring further surgery, was $15.9 \%$ in the PNI-low group and 3.1\% in the PNI-high group. ${ }^{11}$

Similarly, another retrospective study of 556 individuals with colorectal cancer yielded similar results. ${ }^{13}$ Low PNI groups $(<45.5)$ were associated with almost a 2 -fold higher incidence of severe postoperative complications than high PNI groups. Furthermore, low PNI groups were observed to have a tumour recurrence rate of $15.9 \%$ compared with $8.7 \%$ in high PNI groups. ${ }^{13}$

An inverse relationship was observed between lower PNI values and advanced-stage colorectal cancer, increased severity of postoperative complications, and unplanned admission to the intensive care unit. Patients classified as malnourished with a PNI less than 40 also had a prolonged length of stay in hospital compared with well-nourished patients (mean $16.4 \mathrm{~d} \mathrm{v} .9 .2 \mathrm{~d}$ ). ${ }^{12}$ Another retrospective study of 351 patients reported that a low PNI $(<40)$ was associated with higher incidence of surgical site infection. ${ }^{14}$ However, when using PNI to evaluate malnutrition in patients with colorectal cancer, it is important to consider that a low PNI score can be associated with additional factors, such as older age. ${ }^{13}$ Nonetheless, PNI is a valid, accessible, and widely available nutritional assessment. ${ }^{12}$

\section{Nutritional Risk Index}

The NRI measures serum albumin and weight loss and categorizes patients as mildly, moderately, or severely malnourished..$^{8,15}$ The following formula may be used to calculate NRI: $1.489 \times$ serum albumin $(\mathrm{g} / \mathrm{L})+41.7 \times$ (present weight $\div$ usual weight). A score greater than 100 indicates that the patient is not malnourished, 97.5 to 100 indicates mild malnourishment, 83.5 to less than 97.5 moderate malnourishment, and less than 83.5 severe malnourishment. Although there are limited studies clarifying that NRI is an effective predictor of postoperative outcome in patients with colorectal cancer, a study by Sungurtekin and colleagues of 100 patients who had undergone major intra-abdominal surgery showed that NRI, when compared with SGA, was also predictive of malnutrition and postoperative complications in these populations..$^{15}$

When evaluated against the PG-SGA, which served as the gold standard, the NRI had a lower sensitivity (66\%) and specificity (60\%). ${ }^{16}$ Additionally, although the NRI itself is a quick and simple technique, it requires blood biochemical parameter tests. ${ }^{717}$ That being said, its objectivity and potential predictive value allow it to be useful in screening for malnutrition in patients with colorectal cancer.

\section{Malnutrition Universal Screening Tool}

The MUST accounts for a patient's BMI, weight loss during the previous 3-6 months, and periods of acute disease 
longer than 5 days during which they found it almost impossible to eat. These 3 parameters are assigned scores that are then totaled so that the patient may be categorized as being at low (score $=0)$, moderate $($ score $=1)$, or high risk of malnutrition (score $\geq 2$ ). ${ }^{7,8}$ A prospective cohort study of 80 patients found that a score indicating high risk of malnutrition according to MUST was significantly associated with a higher risk of postoperative complications following oncological colorectal resection. ${ }^{18}$

The sensitivity and specificity of MUST were $96 \%$ and $75 \%$, respectively, when SGA was used as the reference standard. ${ }^{8}$ The MUST is a simple and easy to use nutritional screening tool that can be completed by any health professional, and it takes less time to complete than the SGA - only 3-5 minutes. ${ }^{7,8}$

\section{Nutritional Risk Screening 2002}

The NRS-2002 evaluates 2 major components: impaired nutritional status (accounting for weight loss, BMI and amount of food intake) and the severity of the disease. ${ }^{19}$ The patient receives a score of $0-3$ for impaired nutritional status, another score of $0-3$ for severity of the disease, and an age adjustment score of +1 if they are older than 70 years; the scores are then totaled. A total score greater than 3 indicates that a patient is at nutritional risk. ${ }^{19}$ Preoperative NRS-2002 screening has successfully predicted postoperative complication rates and length of hospital stay in patients with colorectal cancer: malnourished patients had significantly higher complication rates and longer hospital stays than those not at nutritional risk. ${ }^{5,19}$ In particular, nutritional risk corresponded with increased wound infection and anastomotic leakage. ${ }^{19}$ However, the NRS-2002 was unable to effectively predict death. ${ }^{5}$ Despite this disadvantage, the NRS-2002 is a simple and efficient tool. ${ }^{5,19}$ Additionally, based on its interobserver variation between physicians and among nurses, dietitians and physicians, the NRS-2002 is a reliable and reproducible tool. . $^{50}$

\section{Reilly's Nutrition Risk Score}

The NRS is a screening tool that incorporates unintentional weight loss in the past 3 months, BMI, appetite, ability to eat and/or retain food, and severity of the disease. A score of $0-3$ is assigned to each of these components, and total score greater than 4 indicates that a patient is at nutritional risk. The NRS was found to be a significant predictor of death among patients with colorectal cancer, although it did not predict morbidity. ${ }^{5}$ The same study, which compared the NRS with the NRS-2002 in 186 patients, found that although both tools were effective, there were discrepancies in their predictive value that may be explained by the factors by which they are evaluated and their risk threshold. The NRS-2002 requires a lower total score to predict morbidities and thus may be a better assessment of perioperative risks of complication or death than the NRS. ${ }^{5}$ However, its simplicity and efficiency render it useful for routine malnutrition screening. Furthermore, because the interobserver variation between dietitians and nursing staff was minimal, this consistency reduces the requirement for dietician referrals, as the assessment may be completed by nursing staff. ${ }^{5,17}$

\section{Serum albumin and prealbumin}

Preoperative serum albumin is an effective predictor of both short-term and long-term outcomes of colon cancer surgery. In the short term, hypoalbuminemic patients (serum albumin $<35 \mathrm{~g} / \mathrm{L}$ ) are reported to have significantly higher rates of postoperative morbidity and mortality, as well as complications related to wounds, lungs, urinary system, and anastomosis compared with patients with normal serum albumin levels. ${ }^{21}$ In the long term, the 5-year overall survival rates and 5-year relapse-free survival rates were significantly poorer in hypoalbuminemic patients than in patients with normal serum albumin levels. ${ }^{21}$

Similarly, a multi-institutional retrospective study comparing serum albumin with weight loss and BMI found that hypoalbuminemia significantly predicted 30 -day mortality and 19 specific postoperative morbidities. ${ }^{1}$ These complications included surgical site infection, wound disruption, pulmonary embolism, acute renal failure, urinary tract infections, septic shock, and return to the operating room. Both weight loss and BMI also predicted 30-day mortality; however, they predicted fewer morbidities. Although weight loss and an underweight status were associated with increased length of hospital stay, hypoalbuminemia still had the strongest association. ${ }^{1}$ Therefore, out of these 3 nutritional status parameters, serum albu$\mathrm{min}$ is the most reliable screening tool for predicting surgical risks and postoperative morbidities in patients with colorectal cancer. However, serum albumin may not be the most effective biochemical marker for predicting acute changes in nutritional status. ${ }^{22}$ Alternatively, prealbumin may be used as it has a shorter half-life than serum albumin, allowing dietitians to better evaluate the effects of short-term nutritional interventions. ${ }^{8,22}$ Although this characteristic allows for the routine use of prealbumin in hospitals, prealbumin is not as effective as serum albumin in predicting short-term reoccurrence in patients with colorectal cancer. ${ }^{23}$ It is also noteworthy that there are several non-nutritional factors, such as systemic inflammation, that influence both serum albumin and prealbumin concentrations, thus low levels of these laboratory markers may not always indicate malnutrition. ${ }^{16,22}$ Nonetheless, serum albumin and prealbumin, as objective and widely available nutritional assessments, have been found to be simple and valuable prognostic tools. ${ }^{10,22,23}$ 


\section{Preoperative nutritional interventions}

Identification of patients with preoperative malnutrition allows providers to stratify patients at risk of malnutritionrelated complications. In addition, these tools help identify the groups of patients who would benefit the most from preoperative nutritional support. There are several nutritional interventions that have been found to improve postoperative outcomes and the prognosis of patients with colorectal cancer.

\section{Trimodal prehabilitation program}

Implementing a 1-month trimodal prehabilitation program that combines nutritional support (nutritional counselling and protein supplementation) and anxiety reduction with moderate exercise was found to improve the postoperative functional recovery of patients with colorectal cancer, as outlined by prospective analysis of a single group pilot study and 2 randomized control trials. ${ }^{24}$ As a prehabilitation program of exercise alone had limited improvement on recovery of functional capacity,,$^{25}$ and a prehabilitation program of whey protein alone improved only perioperative functional capacity, ${ }^{26}$ whey protein supplementation was combined with exercise to synergistically increase protein synthesis and muscle strength. ${ }^{27}$ Anxiety reduction was also incorporated to motivate and reinforce compliance with the exercise and nutritional interventions. As a result, this trimodal prehabilitation program was found to improve postoperative recovery. Based on the 6-minute walk test $(6 \mathrm{MWT})$, which was measured at baseline, preoperatively, and at 4 and 8 weeks postoperatively, $81 \%$ of the prehabilitated patients recovered their baseline $6 \mathrm{MWT}$ by 8 weeks after surgery compared with only $40 \%$ in the control group. ${ }^{27}$

A similar single-blind parallel-arm superiority randomized controlled trial of 77 patients comparing trimodal prehabilitation with trimodal rehabilitation found that by 8 weeks after colorectal cancer surgery, $84 \%$ of the prehabilitated patients recovered to baseline walking capacity compared with only $62 \%$ of the rehabilitated patients. ${ }^{28}$ In fact, at 8 weeks after surgery, on average, the prehabilitated patients were above baseline walking capacity, whereas the rehabilitated patients were below baseline walking capacity. ${ }^{28}$ Intervention at the preoperative period may thus be more effective at improving postoperative recovery as patients are generally in better physical condition than they are in the postoperative period.

Although it is uncertain which intervention of the trimodal program contributes the most to functional capacity, incorporating multimodal prehabilitation has proven to be beneficial, as it is associated with improvements in postoperative recovery in patients with colorectal cancer. ${ }^{24}$

\section{Modified peripheral parenteral nutrition with fat emulsion, multiple vitamins, and trace elements}

Enteral nutrition is insufficient for severely malnourished patients, total parenteral nutrition may be too risky, and peripheral parenteral nutrition (PPN) with fat emulsion may be inadequate in terms of energy and nutrients; therefore, a modified PPN with fat emulsion, multiple vitamins and trace elements was investigated as a possible preoperative nutritional support for patients undergoing colorectal cancer resection. This combined nutritional support was found to have postoperative benefits because patients receiving PPN with fat emulsion, multivitamins, and trace elements had significantly higher serum albumin levels; lower C-reactive protein and white blood cell levels, which are indicative of lower rates of infection and inflammation; lower anastomotic leak rates; and shorter hospital stays than patients receiving only PPN with fat emulsion. ${ }^{4}$ The addition of zinc, a trace element associated with wound healing, may explain why patients receiving only PPN with fat emulsion had increased inflammatory response and delayed wound healing compared to those receiving PPN with fat emulsion, multivitamins and trace elements. ${ }^{4,29}$ The implementation of PPN with fat emulsion, multivitamins and trace elements for 4 days preoperatively in addition to oral or enteral feeding as tolerated was sufficient to see postoperative benefits, demonstrating that improving the preoperative micronutrient status of patients with colorectal cancer for a short period of time can improve their prognosis. ${ }^{4}$

\section{Arginine and N-3 fatty acid immunonutrition}

Malnutrition has been found to decrease immunonutritional status ${ }^{11}$ and, consequently, immunonutrient deficiencies have been shown to cause increased postoperative infectious complications and poor prognosis. ${ }^{30}$ Therefore, preoperative supplementation of immunonutrients should strongly be considered as a best practice in preoperative care for colorectal cancer.

Immunonutrients, most notably arginine and $\mathrm{N}-3$ fatty acids, have been found to decrease postoperative infections. ${ }^{30,31}$ Arginine boosts immunometabolism by improving $\mathrm{T}$ cell function, facilitates wound healing, and stimulates the nitric oxide pathway for vasodilation. ${ }^{32} \mathrm{~N}-3$ fatty acids exert anti-inflammatory effects by maintaining eicosanoid stores. ${ }^{33}$ Markedly, in a study of 100 patients with colorectal cancer, those who reported a habitual dietary intake of $1.2 \mathrm{~g} / \mathrm{d}$ of $\mathrm{N}-3$ fatty acids experienced significantly fewer postoperative complications than those taking only $0.7 \mathrm{~g} / \mathrm{d}$ of N-3 fatty acids. ${ }^{34}$

Another study found that patients receiving supplementation with the aforementioned immunonutrients 
experienced improved immune response. Impact is an immunonutrition supplement containing arginine, $\mathrm{N}-3$ fatty acids, and ribonucleic acids. ${ }^{30,31}$ Patients who received oral Impact either preoperatively or perioperatively reported better immune response, gut oxygenation, and microperfusion than patients in the groups that received either a conventional isonitrogenous formula or no supplementation. In particular, upon supplementation of preoperative immunonutrition, the infection rate in the Impact group was $12 \%$, compared with $30 \%$ and $32 \%$ in the conventional supplementation and control groups, respectively. ${ }^{31}$ In another randomized trial of 60 patients, individuals who received preoperative Impact by a nasal feeding catheter had improved postoperative nutritional status and immunological function compared with those receiving standard enteral nutrition. Additionally, the length of hospital stay and incidence of postoperative infections was significantly lower in patients who received Impact. ${ }^{30}$ These results show the benefits of incorporating immunonutritional supplements in the preoperative regimen of patients with colorectal cancer to improve postoperative prognosis.

\section{Postoperative NUTRITIONAL INTERVENTIONS}

In addition to preoperative nutritional care, the implementation of postoperative nutritional practices may further enhance the recovery and improve the nutritional status of malnourished patients with colorectal cancer. Common postoperative nutritional interventions, such as oral nutritional supplements and total parenteral nutrition, and their beneficial effects on postoperative outcomes in patients with gastrointestinal cancer are discussed in the sections that follow.

\section{Oral nutritional supplements}

Postoperative oral nutritional supplements in conjunction with preoperative supplements have been found to be an effective means of improving postoperative outcomes. ${ }^{35,36}$ A study comparing a retrospectively gathered control group (105 patients) that followed a normal diet with a prospectively gathered group receiving highprotein nutritional support both pre- and postoperatively (52 patients) found that the occurrence of wound dehiscence, anastomosis dehiscence, infection, and rehospitalization was $2.2,4.3,2.0$, and 1.7 times lower, respectively, in the nutrition support group..$^{35}$ The mean length of hospital stay was also reduced in patients receiving oral supplemental nutritional care. ${ }^{35}$ An additional prospective 4-arm double-blind randomized controlled trial of 120 patients showed that when patients were given a carbohydrate drink preoperatively and a polymeric supplement postoperatively, they had improved postoperative handgrip strength, pulmonary function, and insulin resistance compared with patients given placebo supplements. Although patients who received solely preoperative or postoperative oral nutritional supplements still showed postoperative improvements, the effect was weaker. ${ }^{36}$ This stresses the importance of continuity of pre- to postoperative nutritional care to achieve maximal recovery. This study further demonstrated that enhanced recovery was seen when patients consumed smaller amounts of the nutritional supplements than what was prescribed. ${ }^{36}$ Therefore, even minimal amounts of oral nutritional supplements can have a positive impact on postoperative outcome.

\section{Total parenteral nutrition}

Total parenteral nutrition in patients with colorectal cancer has also been highlighted as an important postoperative nutritional practice. The administration of postoperative total parenteral nutrition has been found to decrease the rate of further complications, improve antioxidant capacity, and improve nitrogen balance in cancer patients postoperatively. ${ }^{37,38}$ Patients who received essential branched-chain amino acid support of leucine, isoleucine, and valine via total parenteral nutrition postoperatively were found to have an improved or more positive nitrogen balance despite the stress-induced catabolic state after surgery. ${ }^{37}$ Furthermore, total parenteral nutrition administered in the postoperative period has been shown to alleviate oxidative stress ensuing from gastrointestinal surgery. Patients with colorectal cancer who received total parenteral nutrition were found to have an increase in superoxide dismutase, a key enzyme in the antioxidant pathway, compared with the control group who received standard intravenous fluid therapy. ${ }^{38}$

\section{Discussion}

Owing to the high prevalence of preoperative malnutrition in patients undergoing colorectal cancer surgery, it is essential that malnutrition in these patients is assessed and treated to optimize their recovery. Although further research is required to determine the most useful method of screening malnutrition, SGA, PG-SGA, PNI, NRI, MUST, NRS-2002, NRS, serum albumin, and prealbumin should be considered, as they have been found to be effective predictors of postoperative outcomes. Table 1 summarizes these preoperative nutritional assessments. Their cost, validity, and ease of use should be considered as well. Further studies should also explore additional programs to reduce malnutrition in these patients. Nonetheless, the implementation of preoperative nutritional support must become a priority, and there are several simple yet effective nutritional interventions, such as trimodal prehabilitation, modified peripheral parenteral 


\begin{tabular}{|c|c|c|c|c|c|c|c|c|c|}
\hline $\begin{array}{l}\text { Nutritional } \\
\text { assessment }\end{array}$ & $\begin{array}{l}\text { Requires blood } \\
\text { biochemical } \\
\text { parameter test }\end{array}$ & $\begin{array}{c}\text { Person } \\
\text { performing } \\
\text { assessment }\end{array}$ & $\begin{array}{c}\text { Mode of } \\
\text { assessment }\end{array}$ & $\begin{array}{l}\text { Efficiency of } \\
\text { assessment }\end{array}$ & $\begin{array}{l}\text { Accounts } \\
\text { for weight } \\
\text { loss and/ } \\
\text { or BMI }\end{array}$ & $\begin{array}{c}\text { Requires } \\
\text { physical } \\
\text { assessment }\end{array}$ & $\begin{array}{l}\text { Accounts } \\
\text { for dietary } \\
\text { intake }\end{array}$ & $\begin{array}{l}\text { Accounts for } \\
\text { medical } \\
\text { condition/ } \\
\text { severity of the } \\
\text { disease }\end{array}$ & $\begin{array}{l}\text { Accounts } \\
\text { for } \\
\text { functional } \\
\text { capacity }\end{array}$ \\
\hline SGA & No & $\begin{array}{c}\text { Trained } \\
\text { health care } \\
\text { professional* }^{*}\end{array}$ & $\begin{array}{l}\text { Form (detailed } \\
\text { questionnaire) }\end{array}$ & Efficient & $\begin{array}{l}\text { Weight } \\
\text { loss }\end{array}$ & Yes & Yes & Yes & Yes \\
\hline PG-SGA & No & $\begin{array}{c}\text { Trained } \\
\text { health care } \\
\text { professional* } \\
\text { and patient }\end{array}$ & $\begin{array}{l}\text { Form (2-part } \\
\text { detailed ques- } \\
\text { tionnaire) }\end{array}$ & Efficient & $\begin{array}{l}\text { Weight } \\
\text { loss }\end{array}$ & Yes & Yes & Yes & Yes \\
\hline PNI & $\begin{array}{c}\text { Serum albumin } \\
+ \text { lymphocyte } \\
\text { count }\end{array}$ & $\begin{array}{l}\text { Physician, } \\
\text { dietitian, or } \\
\text { nurse }\end{array}$ & Equation & $\begin{array}{l}\text { Quick but } \\
\text { requires } \\
\text { laboratory } \\
\text { parameter }\end{array}$ & No & No & No & No & No \\
\hline$N R I$ & Serum albumin & $\begin{array}{l}\text { Physician, } \\
\text { dietitian, or } \\
\text { nurse }\end{array}$ & Equation & $\begin{array}{l}\text { Quick but } \\
\text { requires } \\
\text { laboratory } \\
\text { parameter }\end{array}$ & $\begin{array}{l}\text { Weight } \\
\text { loss }\end{array}$ & No & No & No & No \\
\hline MUST & No & $\begin{array}{l}\text { Physician, } \\
\text { dietitian or } \\
\text { nurse }\end{array}$ & $\begin{array}{l}\text { Form (scored } \\
\text { questionnaire) }\end{array}$ & Very efficient & Both & No & Yes & Yes & No \\
\hline NRS-2002 & No & $\begin{array}{c}\text { Physician, } \\
\text { dietitian, or } \\
\text { nurse }\end{array}$ & $\begin{array}{l}\text { Form (scored } \\
\text { questionnaire) }\end{array}$ & Very efficient & Both & No & Yes & Yes & No \\
\hline NRS & No & $\begin{array}{l}\text { Physician, } \\
\text { dietitian, or } \\
\text { nurse }\end{array}$ & $\begin{array}{l}\text { Form (scored } \\
\text { questionnaire) }\end{array}$ & Very efficient & Both & No & Yes & Yes & No \\
\hline $\begin{array}{l}\text { Serum } \\
\text { albumin }\end{array}$ & Yes & $\begin{array}{l}\text { Physician, } \\
\text { dietitian, or } \\
\text { nurse }\end{array}$ & $\begin{array}{l}\text { Laboratory } \\
\text { parameter }\end{array}$ & $\begin{array}{l}\text { Quick but } \\
\text { requires } \\
\text { laboratory } \\
\text { parameter }\end{array}$ & No & No & No & No & No \\
\hline Prealbumin & Yes & $\begin{array}{l}\text { Physician, } \\
\text { dietitian, or } \\
\text { nurse }\end{array}$ & $\begin{array}{l}\text { Laboratory } \\
\text { parameter }\end{array}$ & $\begin{array}{l}\text { Quick but } \\
\text { requires } \\
\text { laboratory } \\
\text { parameter }\end{array}$ & No & No & No & No & No \\
\hline \multicolumn{10}{|c|}{$\begin{array}{l}\text { BMI = body mass index; MUST = Malnutrition Universal Screening Tool; NRI = Nutritional Risk Index; NRS = Reilly's Nutrition Risk Score; NRS-2002 = Nutritional Risk Screening 2002; } \\
\text { PG-SGA = Patient-Generated Subjective Global Assessment; PNI = Prognostic Nutritional Index; SGA = Standard Global Assessment. } \\
\text { *Physician, dietitian, or nurse must be trained to perform assessment. }\end{array}$} \\
\hline
\end{tabular}

Table 2. Summary of preoperative nutritional interventions

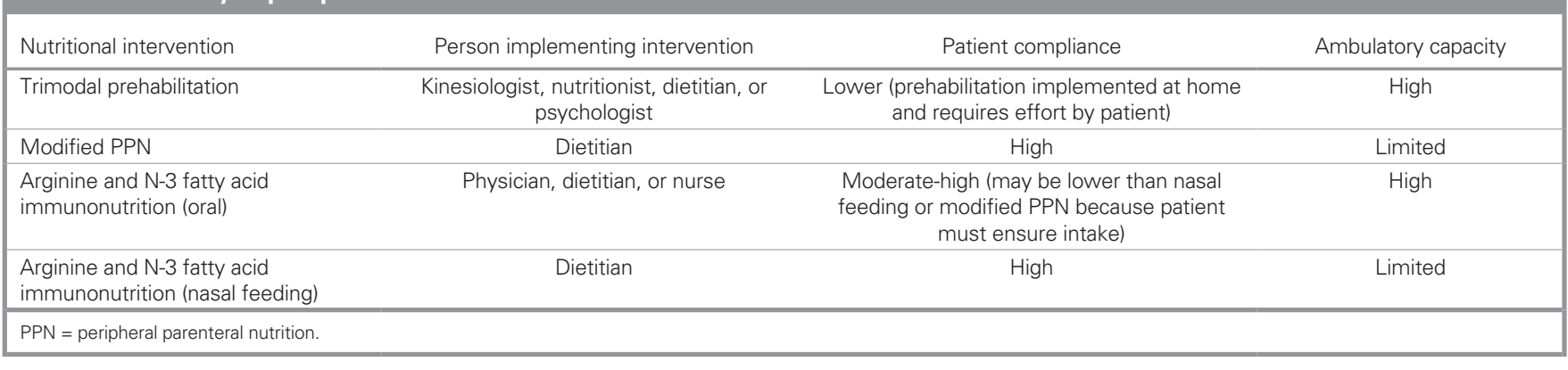

nutrition, and N-3 fatty acid and arginine supplementation, that have benefited patients postoperatively. Table 2 highlights the major characteristics of these preoperative nutritional interventions. Additional studies should also investigate the combined effect of the aforementioned pre- and postoperative nutritional interventions to highlight the importance of maintaining continuity of care throughout a patient's surgical treatment in its entirety.
Determining the settings in which these assessments and treatments may be applied is also noteworthy. An algorithm has been provided in Figure 1, highlighting which assessments and treatments may be performed in an inpatient compared with an outpatient setting. Screening preoperative malnutrition in patients with colorectal cancer and developing preoperative nutritional support programs will decrease postoperative complications and length of hospital 


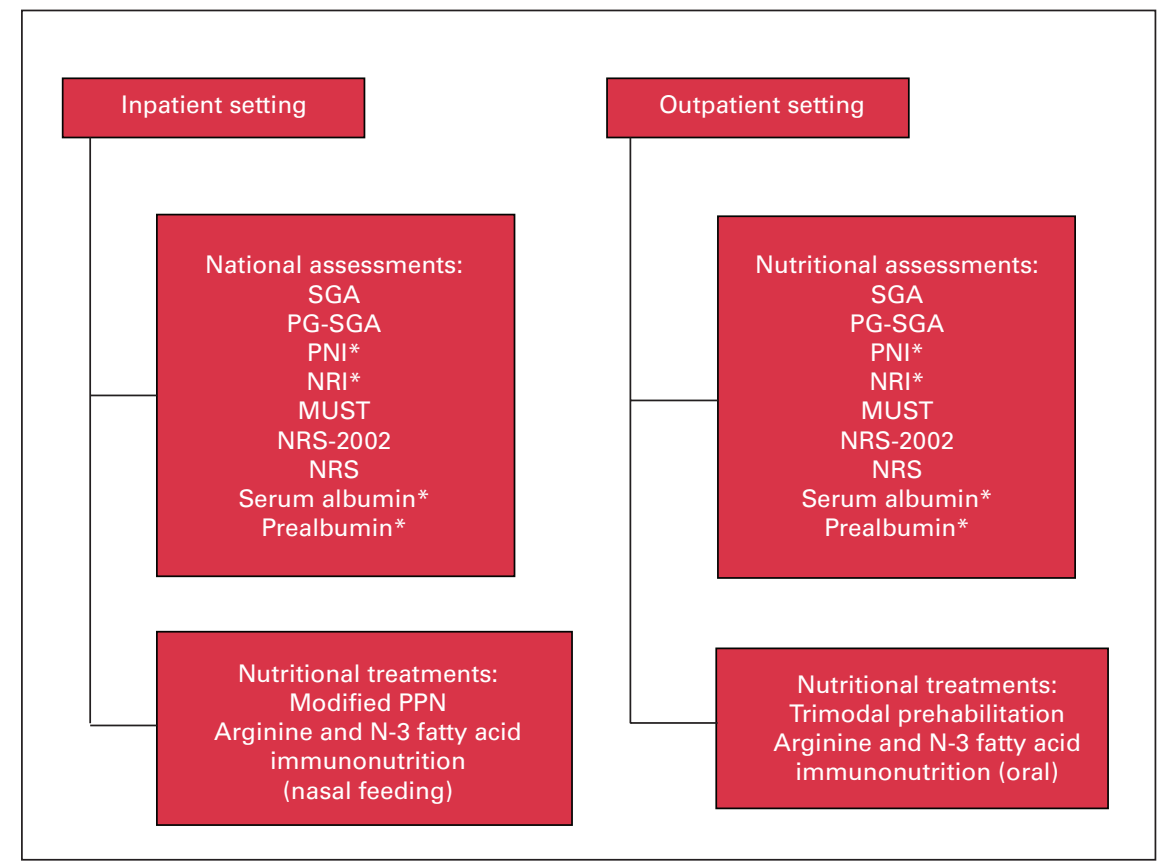

Fig. 1. Preoperative nutritional assessments and treatments categorized by the setting in which they would be conducted. * Requires a blood biochemical parameter test and thus may take longer to complete. Note: MUST = Malnutrition Universal Screening Tool; NRI = Nutritional Risk Index; NRS = Reilly's Nutrition Risk Score; NRS-2002 = Nutritional Risk Screening 2002; PG-SGA = Patient-Generated Subjective Global Assessment; PNI = Prognostic Nutritional Index; SGA = Standard Global Assessment.

stay and improve prognosis, ultimately enhancing overall quality of care.

\section{Conclusion}

Preoperative malnutrition in patients undergoing surgery for colorectal cancer is a common and serious issue that affects postoperative recovery and patient outcomes. Although further research is required to conclusively determine the most useful method of screening malnutrition, we suggest that routine assessment in the preoperative stage in patients with colorectal cancer is essential to achieve the desirable outcome.

Affiliations: From the Department of Surgery, Western University, London, Ont.

\section{Competing interests: None declared.}

Contributors: A. Gupta, E. Gupta, C. Schlachta and N. Alkhamesi designed the study. A. Gupta, E. Gupta and N. Alkhamesi acquired and analysed the data, which R. Hilsden, J. Hawel and A. Elnahas also analysed. A. Gupta, E. Gupta and N. Alkhamesi wrote the article, which all authors reviewed. All authors approved the final version to be published.

Content licence: This is an Open Access article distributed in accordance with the terms of the Creative Commons Attribution (CC BYNC-ND 4.0) licence, which permits use, distribution and reproduction in any medium, provided that the original publication is properly cited, the use is noncommercial (i.e., research or educational use), and no modifications or adaptations are made. See: https://creativecommons. org/licenses/by-nc-nd/4.0/

\section{References}

1. Hu WH, Cajas-Monson L, Eisenstein S, et al. Preoperative malnutrition assessments as predictors of postoperative mortality and morbidity in colorectal cancer: an analysis of ACS-NSQIP. Nutr 7 2015;14:91.

2. Capra S, Ferguson M, Ried K. Cancer: impact of nutrition intervention outcome-nutrition issues for patients. Nutrition 2001;17:769-72.

3. Lohsiriwat $\mathrm{V}$. The influence of preoperative nutritional status on the outcomes of an enhanced recovery after surgery (ERAS) programme for colorectal cancer surgery. Tech Coloproctol 2014;18:1075-80.

4. Liu MY, Tang HC, Hu SH, et al. Influence of preoperative peripheral parenteral nutrition with micronutrients after colorectal cancer patients. Biomed Res Int 2015;2015:535431.

5. Schwegler I, Holzen AV, Gutzwiller JP, et al. Nutritional risk is a clinical predictor of postoperative mortality and morbidity in surgery for colorectal cancer. Br F Surg 2010;97:92-7.

6. Barbosa LR, Lacerda-Filho A, Barbosa LC. Immediate preoperative nutritional status of patients with colorectal cancer: a warning. Arq Gastroenterol 2014;51:331-6.

7. Håkonsen SJ, Pedersen PU, Bath-Hextall F, et al. Diagnostic test accuracy of nutritional tools used to identify undernutrition in patients with colorectal cancer: a systematic review. $7 B I$ Database System Rev Implement Rep 2015;13:141-87.

8. Tu MY, Chien TW, Chou MT. Using a nutritional screening tool to evaluate the nutritional status of patients with colorectal cancer. Nutr Cancer 2012;64:323-30.

9. Bauer J, Capra S, Ferguson M. Use of the scored Patient-Generated Subjective Global Assessment (PG-SGA) as a nutrition assessment tool in patients with cancer. Eur 7 Clin Nutr 2002;56:779-85.

10. Poziomyck AK, Gonçalves Fruchtenicht AV, Kabke GB, et al. Reliability of nutritional assessment in patients with gastrointestinal tumors. Rev Col Bras Cir 2016;43:189-97. 
11. Cao X, Zhao G, Yu T, et al. Preoperative Prognostic Nutritional Index correlates with severe complications and poor survival in patients with colorectal cancer undergoing curative laparoscopic surgery: a retrospective study in a single Chinese institution. Nutr Cancer 2017;69:454-63.

12. Seretis C, Kaisari P, Wanigasooriya K, et al. Malnutrition is associated with adverse postoperative outcome in patients undergoing elective colorectal cancer resections. F buon 2018;23:36-41.

13. Tokunaga R, Sakamoto Y, Nakagawa S, et al. Prognostic Nutritional Index predicts severe complications, recurrence, and poor prognosis in patients with colorectal cancer undergoing primary tumor resection. Dis Colon Rectum 2015;58:1048-57.

14. Sagawa M, Yoshimatsu K, Yokomizo H, et al. Worse preoperative status based on inflammation and host immunity is a risk factor for surgical site infections in colorectal cancer surgery. 7 Nippon Med Sch 2017;84:224-30.

15. Sungurtekin H, Sungurtekin U, Balci C, et al. The influence of nutritional status on complications after major intraabdominal surgery. 7 Am Coll Nutr 2004;23:227-32.

16. Faramarzi E, Mahdavi R, Mohammad-Zadeh M, et al. Validation of nutritional risk index method against patient-generated subjective global assessment in screening malnutrition in colorectal cancer patients. Chin 7 Cancer Res 2013;25:544-8.

17. Reilly HM, Martineau JK, Moran A, et al. Nutritional screeningevaluation and implementation of a simple Nutrition Risk Score. Clin Nutr 1995;14:269-73.

18. Kroft GVD, Bours MJL, Janssen-Heijnen M, et al. Value of sarcopenia assessed by computed tomography for the prediction of postoperative morbidity following oncological colorectal resection: a comparison with the malnutrition screening tool. Clin Nutr ESPEN 2018;24:114-19.

19. Kwag SJ, Kim JG, Kang WK, et al. The nutritional risk is a independent factor for postoperative morbidity in surgery for colorectal cancer. Ann Surg Treat Res 2014;86:206-11.

20. Sorensen J, Kondrup J, Prokopowicz J, et al. EuroOOPS: an international, multicentre study to implement nutritional risk screening and evaluate clinical outcome. Clin Nutr 2008;27:340-9.

21. Lai CC, You JF, Yeh CY, et al. Low preoperative serum albumin in colon cancer: a risk factor for poor outcome. Int 7 Colorectal Dis 2011;26:473-81.

22. Truong A, Hanna MH, Moghadamyeghaneh Z, et al. Implications of preoperative hypoalbuminemia in colorectal surgery. World 7 Gastrointest Surg 2016;8:353-62.

23. Fujii T, Sutoh T, Morita H, et al. Serum albumin is superior to prealbumin for predicting short-term recurrence in patients with operable colorectal cancer. Nutr Cancer 2012;64:1169-73.

24. Minnella EM, Bousquet-Dion G, Awasthi R, et al. Multimodal prehabilitation improves functional capacity before and after colorectal surgery for cancer: a five-year research experience. Acta Oncol 2017;56:295-300.
25. Carli F, Charlebois P, Stein B, et al. Randomized clinical trial of prehabilitation in colorectal surgery. Br 7 Surg 2010;97:1187-97.

26. Gillis C, Loiselle SE, Fiore JF, et al. Prehabilitation with whey protein supplementation on perioperative functional exercise capacity in patients undergoing colorectal resection for cancer: a pilot double-blinded randomized placebo-controlled trial. 7 Acad Nutr Diet 2016;116:802-12.

27. Li C, Carli F, Lee L, et al. Impact of a trimodal prehabilitation program on functional recovery after colorectal cancer surgery: a pilot study. Surg Endosc 2013;27:1072-82.

28. Gillis C, Li C, Lee L, et al. Prehabilitation versus rehabilitation: a randomized control trial in patients undergoing colorectal resection for cancer. Anesthesiology 2014;121:937-47.

29. Landsdown ABG, Mirastschijski U, Stubbs N, et al. Zinc in wound healing: theoretical, experimental, and clinical aspects. Wound Repair Regen 2007;15:2-16.

30. Xu J, Zhong Y, Jing D, et al. Preoperative enteral immunonutrition improves postoperative outcome in patients with gastrointestinal cancer. World 7 Surg 2006;30:1284-9.

31. Braga M, Gianotti L, Vignali A, et al. Preoperative oral arginine and n-3 fatty acid supplementation improves the immunometabolic host response and outcome after colorectal resection for cancer. Surgery 2002;132:805-14.

32. Daly JM, Reynolds J, Thom A, et al. Immune and metabolic effects of arginine in the surgical patient. Ann Surg 1988;208:512-23.

33. Gianotti L, Braga M, Fortis C, et al. A prospective, randomized clinical trial on perioperative feeding with an arginine-, omega-3 fatty acid-, and RNA-enriched enteral diet: effect on host response and nutritional status. FPEN 7 Parenter Enteral Nutr 1999;23:314-20.

34. Berstad P, Haugum B, Helgeland M, et al. Preoperative body size and composition, habitual diet, and post-operative complications in elective colorectal cancer patients in Norway. 7 Hum Nutr Diet 2013;26:359-68.

35. Manásek V, Bezdek K, Foltys A, et al. The impact of high protein nutritional support on clinical outcomes and treatment costs of patients with colorectal cancer. Klin Onkol 2016;29:351-357.

36. Lidder P, Thomas S, Fleming S, et al. A randomized placebocontrolled trial of preoperative carbohydrate drinks and early postoperative nutritional supplement drinks in colorectal surgery. Colorectal Dis 2013;15:737-45.

37. Szpetnar M, Matras P, Boguszewska-Czubara A, et al. Is additional enrichment of diet in branched-chain amino acids or glutamine beneficial for patients receiving total parenteral nutrition after gastrointestinal cancer surgery? Adv Clin Exp Med 2014;23:423-31.

38. Szpetnar M, Matras P, Kiełczykowska M, et al. Antioxidants in patients receiving total parenteral nutrition after gastrointestinal cancer surgery. Cell Biochem Funct 2012;30:211-6. 